\title{
Patient-Ventilator Asynchrony in a Traumatically Injured Population
}

\author{
Bryce RH Robinson MD, Thomas C Blakeman MSc RRT, Peter Toth MD, \\ Dennis J Hanseman PhD, Eric Mueller PharmD, and Richard D Branson MSc RRT FAARC
}

\begin{abstract}
BACKGROUND: Prolonged mechanical ventilation, longer hospital stay, and a lower rate of home discharge have been reported with patient-ventilator asynchrony in medical patients. Though commonly encountered, asynchrony is poorly defined within the traumatically injured population. METHODS: Mechanically ventilated trauma patients at an urban, level-1 center were enrolled. Breath waveforms were recorded over 30 min within the first 48 hours following intubation. Asynchronous breaths were defined as ineffective patient triggering, double-triggering, short-cycle breaths, and long-cycle breaths. Asynchronous subjects were defined as having asynchrony in $\geq 10 \%$ of total breaths. Demographic, injury, sedation/delirium scores, and clinical and discharge outcomes were prospectively collected. RESULTS: We enrolled 35 subjects: median age $47 \mathrm{y}, 77.1 \%$ male, $28.6 \%$ with penetrating injuries, $16 \%$ with a history of COPD, median (IQR) Injury Severity Score 22 (17-27), and median (IQR) chest Abbreviated Injury Scale score 2 (0-6). We analyzed $\mathbf{1 5 , 4 4 5}$ breaths. Asynchrony was present in $25.7 \%$ of the subjects. No statistical differences between the asynchronous and non-asynchronous subjects were found for age, sex, injury mechanism, COPD history, delirium/sedation scores, $\mathrm{P}_{\mathrm{aO}_{2}} / \mathrm{F}_{\mathrm{IO}_{2}}$, PEEP, blood gas values, or sedative, narcotic, or haloperidol use. Asynchronous subjects more commonly used synchronized intermittent mandatory ventilation (SIMV) $(100 \%$ vs $38.5 \%, P=.002)$ and took fewer median spontaneous breaths/min: 4 breaths/min (IQR 3-8 breaths/min) vs 12 breaths/min (IQR 9-14 breaths/min) $(P=.007)$. SIMV with set breathing frequencies of $\geq 10$ breaths/min was associated with increased asynchrony rates $(85.7 \%$ vs $14.3 \%, P=.02)$. We found no difference in ventilator days, ICU or hospital stay, percent discharged home, or mortality between the asynchronous and non-asynchronous subjects. CONCLUSIONS: Ventilator asynchrony is common in trauma patients. It may be associated with SIMV with a set breathing frequency of $\geq 10$ breaths/min, though not with longer mechanical ventilation, longer stay, or discharge disposition. (ClinicalTrials.gov NCT01049958) Key words: mechanical ventilation; asynchrony; trauma; critical care. [Respir Care 2013;58(11):1847-1855. (C) 2013 Daedalus Enterprises]
\end{abstract}

\section{Introduction}

Patient-ventilator asynchrony is a common occurrence in critically ill ICU patients. Asynchrony can be defined as a mismatch between the patient's ventilator needs and the ventilator settings and operating characteristics. ${ }^{1-5}$ Studies

\footnotetext{
The authors are affiliated with the Division of Trauma and Critical Care, Department of Surgery, University of Cincinnati College of Medicine, Cincinnati, Ohio.

Mr Branson has disclosed relationships with Ikaria, Advanced Circulatory Systems, and General Electric. The other authors have disclosed no conflicts of interest.
}

have shown that asynchrony may be associated with a longer duration of mechanical ventilation, due to ineffec-

\footnotetext{
Dr Robinson presented a version of this paper at the Critical Care Congress of the Society of Critical Care Medicine, held February 4-8, 2012, in Houston Texas.

Correspondence: Bryce RH Robinson MD, Division of Trauma and Critical Care, Department of Surgery, University of Cincinnati College of Medicine, 231 Albert Sabin Way, Cincinnati OH 45267-0558. E-mail: bryce.robinson@uc.edu.
}

DOI: $10.4187 /$ respcare. 02237 
tive weaning, resulting in longer ICU and hospital stay, and worse outcomes. ${ }^{1,6}$

Asynchrony has been evaluated in a number of studies that reviewed the pressure, volume, and flow waveforms from the mechanical ventilator. ${ }^{1-5}$ This technique has limitations, including the ability to visualize the waveforms on a variety of different ventilators with different display resolutions, reliance on the internal measurement systems of the ventilator, and lack of traditional esophageal pressure monitoring. Nonetheless, previous work has demonstrated the validity of ventilator waveforms evaluation, compared to esophageal pressure monitoring, for the detection of missed triggers and asynchrony. ${ }^{1}$ Additionally, these waveforms are commonly utilized by the clinical staff both to detect and to alleviate asynchrony at the bedside of patients experiencing respiratory distress.

See the Related Editorial on Page 1992

Traumatically injured patients differ greatly from previously investigated populations, by virtue of lower age with resultant decreased comorbidities; nonetheless, bedside clinicians encounter asynchrony. To date there have been no prospective studies of asynchrony in trauma patients, so the application of previous conclusions made in medical patients appears to be speculative. We conducted a prospective cohort study to determine the frequency and characteristics of asynchrony in a traumatically injured patient population. We hypothesized that asynchrony is present in trauma patients, though the characteristics and implications upon outcomes are different than in medically ill patients.

\section{Methods}

\section{Patient Population}

All traumatically injured patients in the surgical ICU of the University Hospital of the University of Cincinnati who had spontaneous respiratory efforts within 48 hours of initiation of mechanical ventilation were screened. The University Hospital of the University of Cincinnati is an adult American College of Surgeons verified level-1 trauma center that serves 1.8 million people in southwestern Ohio, northern Kentucky, and southeastern Indiana. The hospital is a 693-bed facility with over 100 critical care beds, 34 of which are dedicated to injured and critically ill surgical patients. Exclusion criteria for study participation were age $<18$ years old; patients without spontaneous respirations due to injury, sedation, or chemical paralysis; patients with air leaks in the ventilator circuit such to preclude accurate data collection of pressure-time, flow-time, and tidal volume-time waveforms; and use of a ventilator

\section{QUICK LOOK}

\section{Current knowledge}

Patient-ventilator asynchrony is common in patients with chronic air-flow obstruction. The most common asynchrony during invasive ventilation is missed triggers. An asynchrony index $>10 \%$ is associated with prolonged mechanical ventilation and higher mortality.

\section{What this paper contributes to our knowledge}

Asynchrony is also common in trauma patients, but missed triggers are infrequent because few of these patients have COPD. In trauma patients an asynchrony index $>10 \%$ was not associated with prolonged mechanical ventilation.

other than the Dräger Evita XL (Dräger Medical, Telford, Pennsylvania). The presence or absence of asynchrony was not a criterion for study inclusion. Ventilator-patient waveforms were not collected before study participation. The institutional review board of the University of Cincinnati approved the study; informed consent was obtained from each subject's legally authorized representative. This study was registered with ClinicalTrials.gov (NCT01049958).

\section{Data Acquisition}

Real-time pressure, flow, and volume waveforms were acquired and recorded over $30 \mathrm{~min}$ by a laptop computer connected to the RS 232 output port of the ventilator. Recordings were done once per subject during the first 48 hours of ventilator initiation. None of the subjects had a tracheostomy. Ventilator settings were determined by the clinicians caring for the subjects, and were not manipulated during the recording period. Respiratory therapists provided usual care during the period of recording, and all subjects used a heat and moisture exchanger. Recordings were performed by study personnel during periods in which healthcare delivery did not preclude investigation. Waveforms were analyzed at a later date utilizing VentView software (Dräger Medical, Telford, Pennsylvania).

\section{Asynchrony Criteria}

Asynchronous breaths included ineffective-triggered breaths, double-triggered breaths, short-cycle breaths, and prolonged-cycle breaths. ${ }^{1,6-8}$ Ineffective triggering was defined as a simultaneous decrease in airway pressure and an increase in air flow, without an assisted breath (a wasted patient effort) (Fig. 1). Double-triggered breaths occur when 

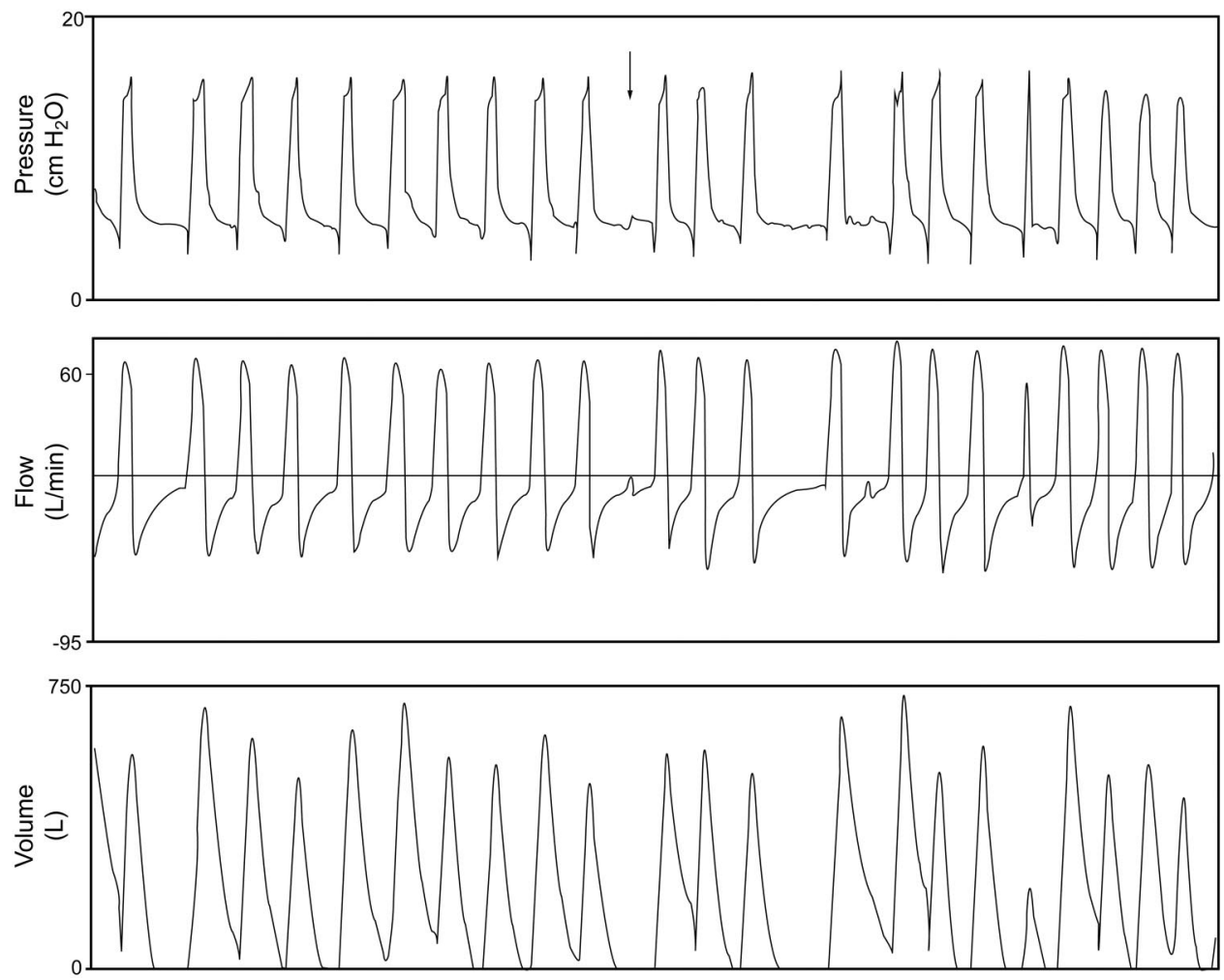

Fig. 1. Missed/ineffective triggering (arrow) is characterized by a decrease in airway pressure and an increase in air flow, without an assisted breath. The ventilator settings were pressure support ventilation with $\Delta 10 \mathrm{~cm} \mathrm{H}_{2} \mathrm{O}$ over $5 \mathrm{~cm} \mathrm{H}_{2} \mathrm{O}$ PEEP.

the ventilator inspiratory time is shorter than the patient's inspiratory time, causing the incomplete patient effort of the first cycle to trigger a second ventilator breath (Fig. 2). Short-cycle breaths were defined as a cycle in which the inspiratory time is less than half the mean set inspiratory time (Fig. 3). Prolonged-cycle breaths describe breaths in which the inspiratory time is more than double the mean set inspiratory time (Fig. 4). A unique asynchronous breath type was identified during the study and labeled as ventilator breath stacking during synchronized intermittent mandatory ventilation (SIMV). This ventilator breath stacking was defined as breaths in which a mandatory breath was delivered during the inspiratory phase of a spontaneous breath (with or without pressure support) (Fig. 5).

Individual pressure, flow, and volume recorded waveforms were reviewed simultaneously by 2 investigators blinded to demographic and outcome data. A third investigator was utilized in circumstances of non-agreement, with agreement between all evaluators necessary before classification as an asynchronous event. The total breaths, including non-asynchronous and asynchronous breaths (ineffective-triggered, double-triggered, short-cycle, prolonged-cycle, and ventilator breath stacking), were calculated for each subject. The asynchrony index was calculated as:
Asynchrony index = number of asynchronous events/ total number of breaths

Subjects with an asynchrony index $\geq 10 \%$ were defined as asynchronous. ${ }^{1,8}$

\section{Subject Characteristics}

Subject demographics, including age, sex, mechanism of traumatic injury, Injury Severity Score, chest Abbreviated Injury Score, smoking history, and history of COPD were recorded.${ }^{9}$ Ventilator settings and arterial blood gas values prior to the initiation of the recording period were noted. Assessment of sedation level and delirium was performed by bedside ICU nurses versed in the Richmond Agitation and Sedation Scale (RASS) and the Confusion Assessment Method in the ICU (CAM-ICU), in adherence to a previously published ICU sedation protocol. ${ }^{10-12}$ Protocolized scoring of pain (via visual analog score), sedation (via RASS), and delirium (via CAM-ICU) occurred every 8-hours or when a change in behavior necessitated an intervention. Predetermined bolus dosing of sedatives and/or analgesics occurred first, in response to objective score changes, with an increase in hourly rates of those mediations if bolus therapy failed. The treatment of delirium with haloperidol occurred only via bolus therapy, 

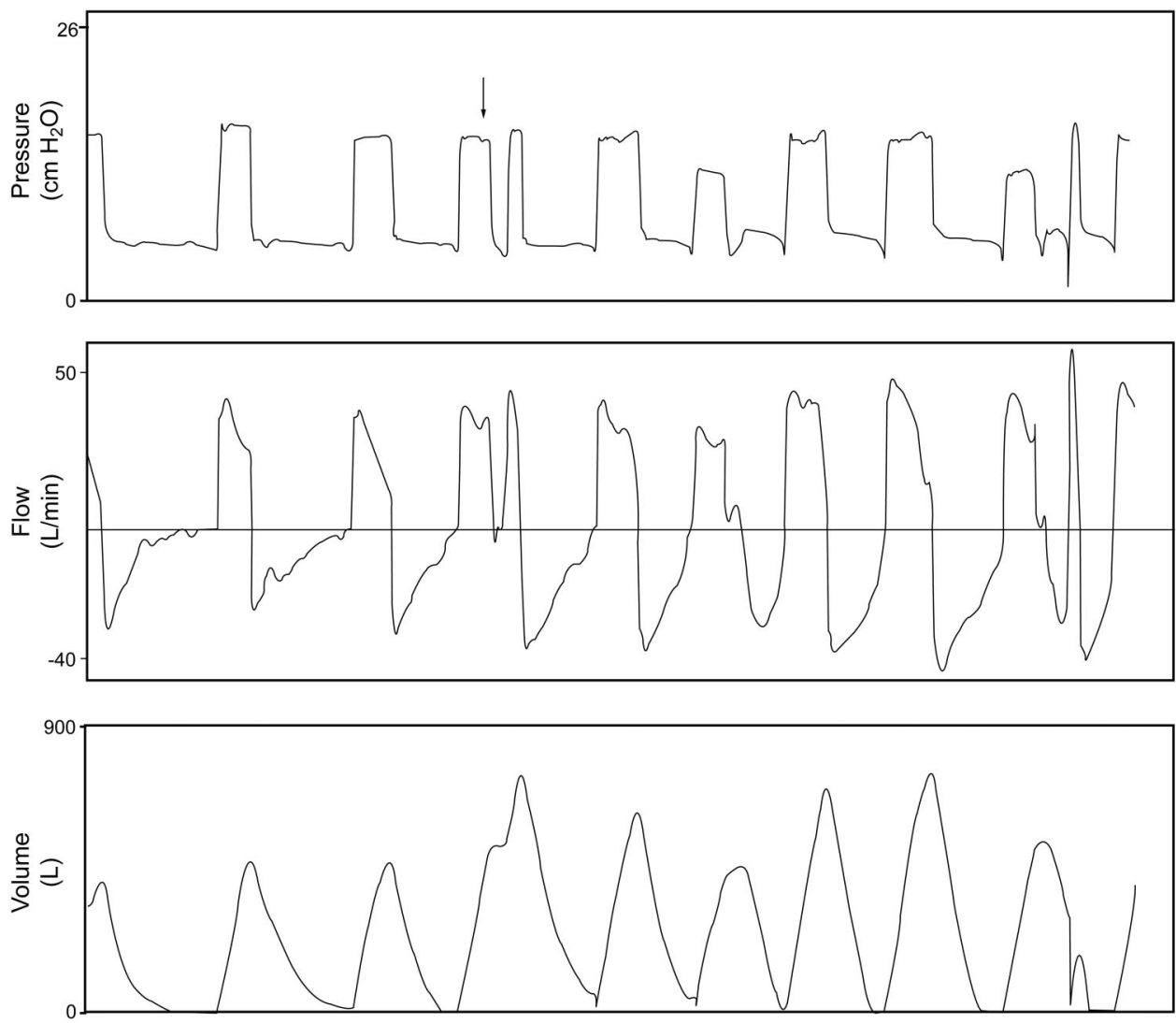

Fig. 2. Double-triggering (arrow) is the result of the ventilator inspiratory time being shorter than the patient's inspiratory time, causing the incomplete patient effort of the first cycle to trigger a second ventilator breath. The ventilator settings were synchronized intermittent mandatory ventilation plus pressure support with $\Delta 10 \mathrm{~cm} \mathrm{H}_{2} \mathrm{O}$ over $5 \mathrm{~cm} \mathrm{H} \mathrm{H}_{2} \mathrm{OEEP}$, with a set frequency of 12 breaths/min. Mandatory breaths are adaptive pressure breaths (AutoFlow mode).

though the frequency of therapy may increase if delirium increases. Propofol and fentanyl were the sedative and analgesic of choice within the first 72 hours after intubation. The RASS and CAM-ICU scores were recorded immediately prior to waveform recording. The amount of fentanyl $(\mu \mathrm{g} / \mathrm{kg})$, propofol $(\mathrm{mg} / \mathrm{kg})$, and haloperidol $(\mathrm{mg})$ for the previous 24 hours and the 1 hour prior to recording were documented.

\section{Data Analysis}

The primary outcome was determined to be the number of ventilator days in subjects with asynchrony index $\geq 10 \%$ versus those with asynchrony index $<10 \%$. Secondary outcomes of ICU stay, hospital stay, proportion of subjects discharged home, and mortality were analyzed between the asynchrony index groups.

Continuous data (non-normally distributed) are summarized using median and IQR, while categorical data are summarized using frequencies and percents. Continuous data were compared between groups using Wilcoxon ranksum tests. Categorical data were compared using exact chi-square tests. All tests were 2 -sided, and $P \leq .05$ was considered statistically significant. Analysis was carried out using statistics software (SAS 9.2, SAS Institute, Cary, North Carolina).

\section{Results}

Seventy trauma patients were screened, 35 were ineligible (20 without spontaneous respirations, 12 for whom consent could not be obtained, and 3 refused participation), and 35 were enrolled in the study (Fig. 6). The study cohort had a median age $47 \mathrm{y}, 77.1 \%$ male, $28.6 \%$ with penetrating injuries, $16 \%$ with a history of COPD, median (IQR) Injury Severity Score 22 (17-27), and median (IQR) chest Abbreviated Injury Scale score $2(0-6)$. The subjects were studied for $30 \mathrm{~min}$ each, yielding a total of 15,445 breath waveforms for analysis. None of the subjects experienced hemodynamic instability or required inotropic and/or vasopressor pharmacotherapy during waveform collection. Twenty-six subjects $(74.3 \%)$ had an asynchrony index $<10 \%$, and $9(25.7 \%)$ had an asynchrony index $\geq 10 \%$. The demographics of the 2 cohorts 


\section{Patient-Ventilator Asynchrony in a Traumatically Injured Population}
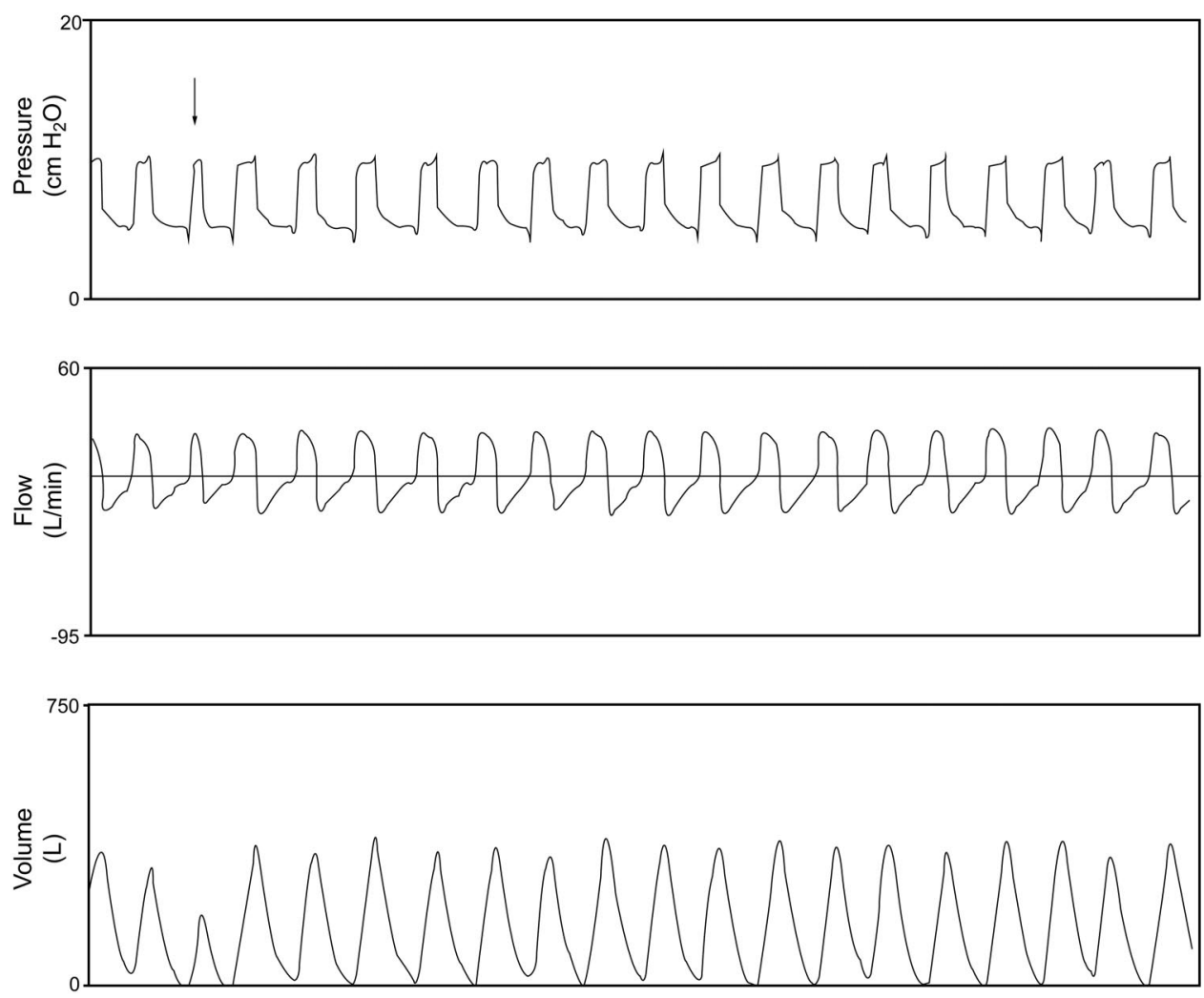

Fig. 3. A short-cycle breath (arrow) has an inspiratory time less than half the mean inspiratory time. Ventilator settings were pressure support ventilation with $\Delta 10 \mathrm{~cm} \mathrm{H}_{2} \mathrm{O}$ over $5 \mathrm{~cm} \mathrm{H}_{2} \mathrm{O}$ PEEP.

are presented in Table 1 . No significant differences were seen in median age, Injury Severity Score, Abbreviated Injury Scale score, proportion of male subjects, rate of penetrating injuries, history of smoking or COPD, $\mathrm{pH}, \mathrm{P}_{\mathrm{aO}_{2}} / \mathrm{F}_{\mathrm{IO}_{2}}$, $\mathrm{P}_{\mathrm{aCO}}$, or bicarbonate between the groups (see Table 1).

When ventilator characteristics were analyzed, subjects with an asynchrony index $\geq 10 \%$ had statistically greater use of SIMV (volume control) plus pressure support ventilation (SIMV + PSV) $(100 \%$ vs $38.5 \%, P=.002)$, with a resultant higher median (IQR) set rate of ventilator breaths/min (12 breaths/min [8-14 breaths/min] vs 0 breaths/min $[0-6$ breaths/min], $P<.001)$ and fewer median (IQR) spontaneous breaths/min (4 breaths/min [38 breaths/min] vs 12 breaths/min [9-14 breaths/min], $P=.007$, Table 2). SIMV + PSV with a set breathing frequency of $\geq 10$ breaths/min was significantly more prevalent in the asynchrony index $\geq 10 \%$ cohort $(85.7 \%$ vs $14.3 \%, P=.02$ ).

The proportions of subjects with a RASS score between -1 and 1 and positive CAM-ICU evaluation were similar between the groups (see Table 2). No difference was found in the use of propofol, fentanyl, or haloperidol. No haloperidol was provided to any subject up to one hour immediately prior to recording.
The $\geq 10 \%$ asynchrony index cohort had 10,896 total breaths (Table 3), of which 1,184 were asynchronous. The $<10 \%$ asynchrony index group had 4,549 breaths, and 235 were asynchronous. Significantly different patterns of asynchronous types were seen in the 2 groups $(P<.01)$ : the $\geq 10 \%$ asynchrony index cohort had more prolongedcycle breaths $(54.7 \%$ vs $11.5 \%)$ and less ventilator breath stacking ( $26.2 \%$ vs $54.9 \%)$. Since ventilator breath stacking has not been among previously described asynchrony breath types, secondary statistical analysis was performed excluding the ventilator breath stacking breaths, and the proportion of asynchrony within the cohort did not significantly differ.

Subjects with an asynchrony index $\geq 10 \%$ had no significant outcome differences (Table 4). Ventilator days, ICU stay, hospital stay, proportion of subjects discharged home, and mortality were similar between the cohorts. The inclusion of long-cycle and short-cycle breaths as asynchronous subtypes may have altered outcome interpretation, so secondary analyses were performed removing those episodes. Without those breaths, 29 subjects had an asynchrony index of $<10 \%$, and 6 subjects had an asynchrony index $\geq 10 \%$. The demographic and arterial blood gas data were not statistically different between the groups. The ventilator settings and proportion of those with SIMV 

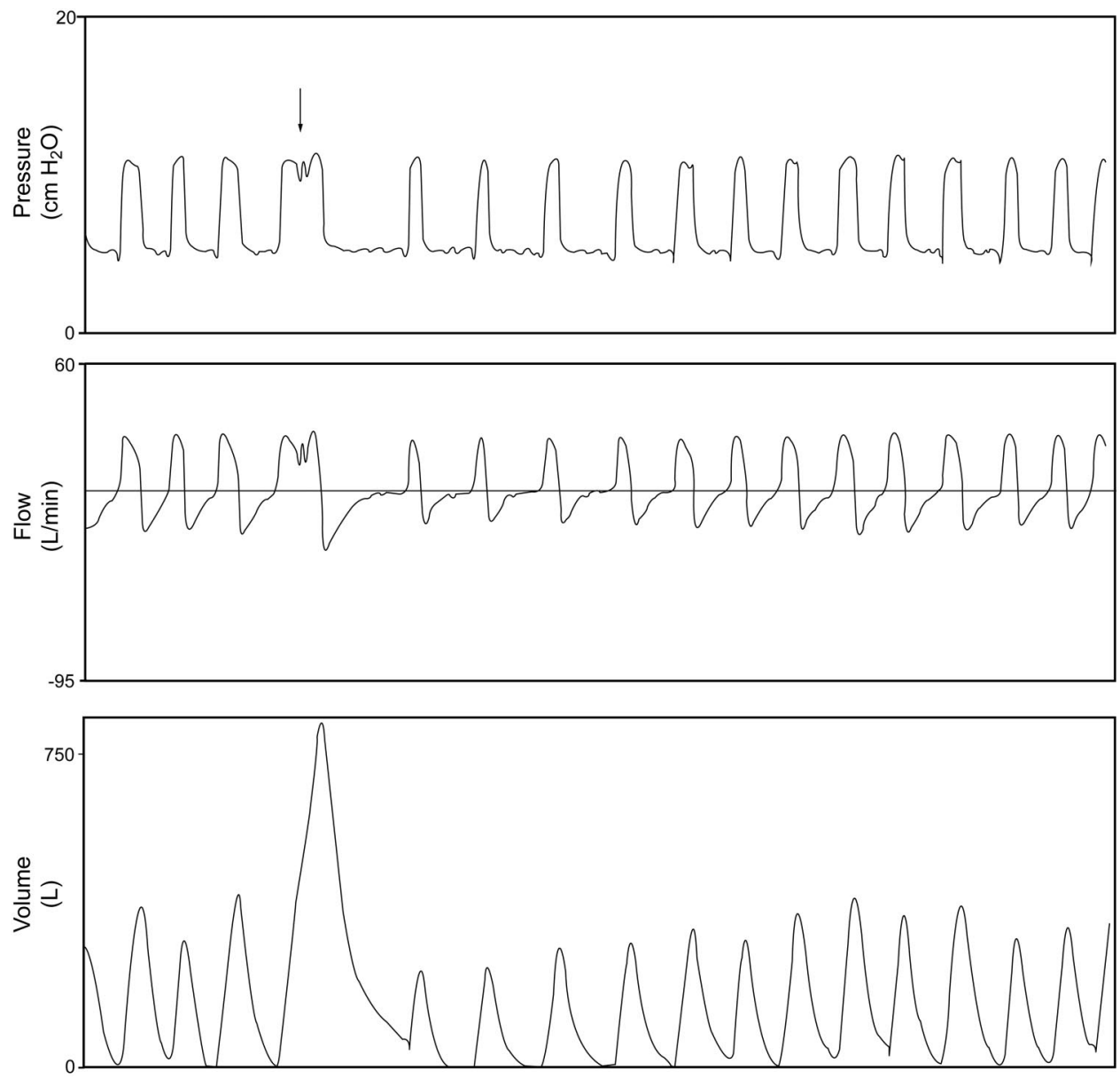

Fig. 4. A prolonged-cycle breath (arrow) has a ventilator inspiratory time more than double the patient's inspiratory time. Ventilator settings were pressure support ventilation with $\Delta 10 \mathrm{~cm} \mathrm{H}_{2} \mathrm{O}$ over $5 \mathrm{~cm} \mathrm{H} \mathrm{H}_{2} \mathrm{O}$ PEEP.

with a ventilator rate $\geq 10$ breaths/min were not different between the groups, though the difference in breathing frequency became nonsignificant. The amount of fentanyl and propofol, and the RASS and CAM-ICU scores were also not statistically different. The proportions of asynchronous breath types were statistically different $(P<.001)$ between those with asynchrony indexes of $<10 \%$ versus $\geq 10 \%$. Ventilator breath stacking accounted for the majority of asynchronous breaths in the $<10 \%$ cohort $(59.7 \%)$, while missed/ ineffective triggers made up the majority in the $\geq 10 \%$ cohort $(43.8 \%)$. There were no statistically significant differences in ventilator days, ICU stay, hospital stay, proportion discharged home, or mortality between the groups after the long-cycle and short-cycle asynchronies were removed.

\section{Discussion}

In this study we investigated the complex interaction of mechanical ventilation with a traumatically injured population so to clarify the presence, etiology and outcomes from patient-ventilator asynchrony. We demonstrated that approximately a quarter of traumatically injured subjects exhibit ventilator asynchrony (asynchrony index $\geq 10 \%$ ) and that these subjects have an associated greater utilization of SIMV + PSV with a set rate of $\geq 10$ breaths $/ \mathrm{min}$. Unique to this work is the finding of ventilator breath stacking (a mandatory breath delivered during the inspiratory phase of a spontaneous breath) as an additional asynchronous breath type. Nonetheless, the presence of asynchrony was not associated with prolonged mechanical ventilation, ICU stay, hospital stay, or mortality. It is noteworthy that, while ineffective triggers are the most common asynchrony in medical patients, missed triggers were relatively uncommon in our subjects. This is likely due to the presence of chronic lung disease and air-trapping in prior studies, which complicate triggering in the medical patient. In a number of our subjects, intrinsic PEEP was present, but, in the absence of dynamic hyperinflation and respiratory muscle dysfunction, did not result in missed triggers.

Patient-ventilator asynchrony is a common problem and is associated with poor outcomes. Chao and StearnHassenpflug demonstrated in 174 chronically ventilator- 

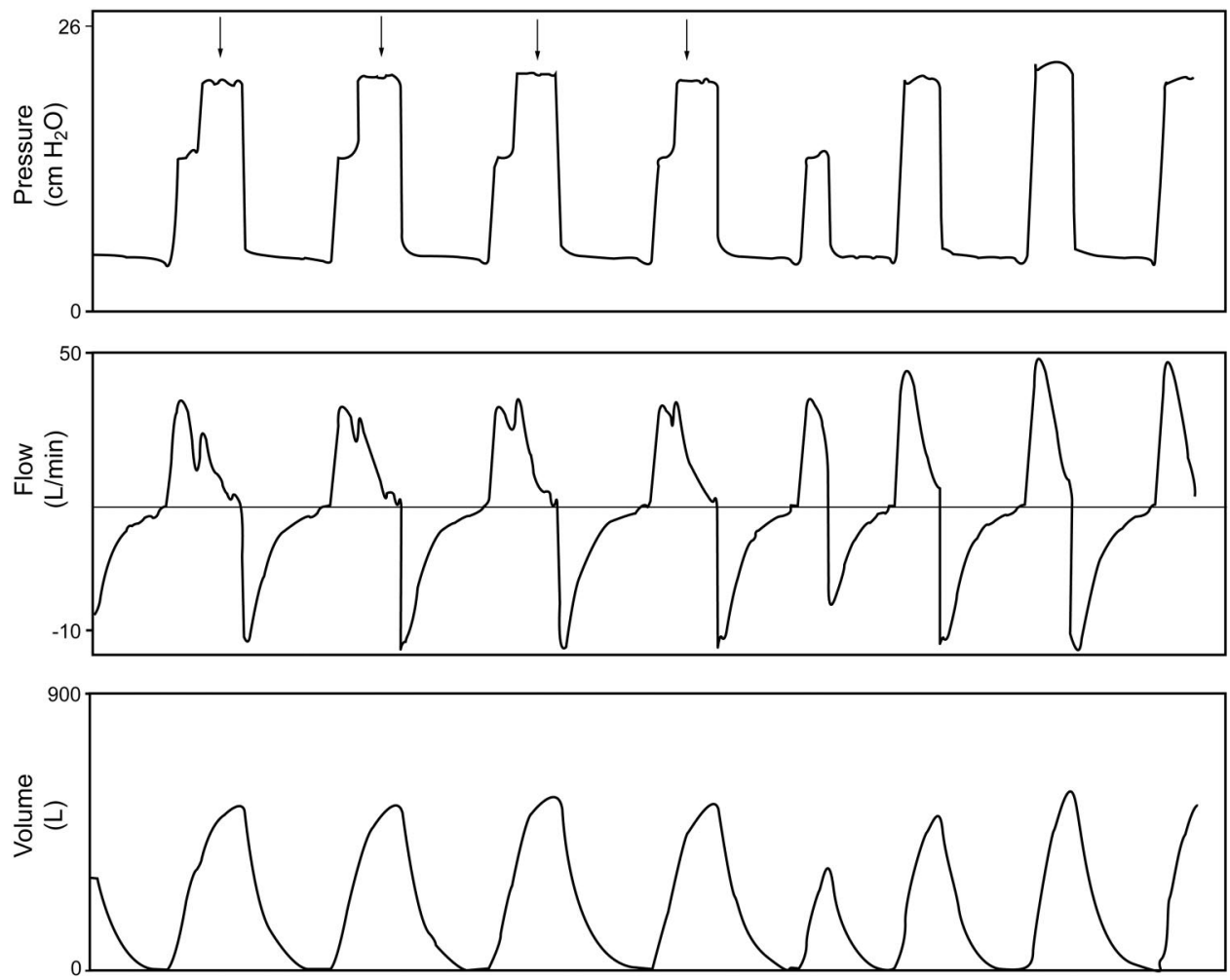

Fig. 5. Ventilator breath stacking (arrows) occurs when a mandatory breath is delivered during the inspiratory phase of a spontaneous breath. Ventilator settings were synchronized intermittent mandatory ventilation plus pressure support (with AutoFlow mode) with $\Delta 10 \mathrm{~cm} \mathrm{H}_{2} \mathrm{O}$ over $5 \mathrm{~cm} \mathrm{H}_{2} \mathrm{O}$ PEEP, with a set frequency of 12 breaths/min. Mandatory breaths are adaptive pressure breaths (AutoFlow mode).

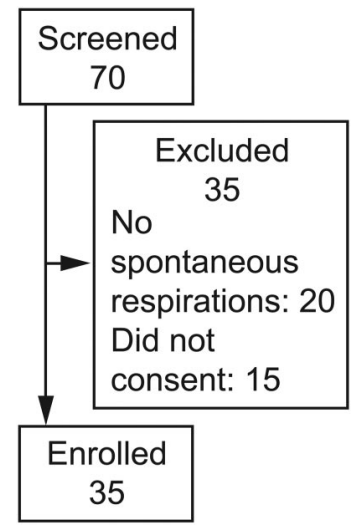

Fig. 6. Enrollment process.

dependent patients that those with ineffective-trigger breaths had longer mechanical ventilation (70 d vs $33 \mathrm{~d}$ ) and a lower rate of eventual liberation ( $16 \%$ vs $57 \%){ }^{6}$ Further work by Thille et al reaffirmed an association of prolonged ventilation with an asynchrony index $\geq 10 \%$ (median duration $25 \mathrm{~d}$ vs $9 \mathrm{~d}$ ), as well as describing a higher rate of tracheostomy (33\% vs 4\%). ${ }^{1}$ However, Chao and Stearn-Hassenpflug studied older critically ill medical patients (69-75 y old), and 45\% had COPD, while Thille et al studied ICU patients, and a quarter had COPD. Inef- fective triggering/missed triggering breaths have been the most common (85-88\%) asynchronous breaths in previous medical studies. ${ }^{1,8}$ These breaths are associated with intrinsic PEEP resulting from large tidal volumes and the continuation of mechanical inspiration during patientdefined expiration. ${ }^{3,4,13}$ Patients with COPD have a higher rate of ineffective triggering; the pressure support level increases because higher patient-generated pressure is required to overcome the intrinsic PEEP associated with hyperinflation and shortened expiratory time..$^{3,6,7,14-16}$

Little is known about patient-ventilator asynchrony in a traumatically injured patients. In comparison to a medically ill population, our study trauma population was younger (median 47 years), with a resultant decreased rate of COPD $(16.1 \%)$. Even with these inherent differences, the proportion of asynchronous subjects (25.7\%) was comparable to previous studies, even in the presence of light sedation goals and delirium monitoring and treatment. ${ }^{1,8}$ Our subjects exhibited unique proportions of asynchrony breath types. With a decreased rate of COPD in our subjects, ineffective triggering occurred infrequently ( $10.1 \%$ of asynchronous breaths), while prolonged-cycle breaths $(54.7 \%)$ were the most common asynchronous breath type in those with $\geq 10 \%$ asynchrony index.

Upon further examination of the prolonged-cycle breaths, we discovered that in the $\geq 10 \%$ asynchrony index group, 


\section{Patient-Ventilator Asynchrony in a Traumatically Injured Population}

Table 1. Demographics and Arterial Blood Gases

\begin{tabular}{lccc}
\hline \hline & $\begin{array}{c}\text { Asynchrony Index } \\
<10 \% \\
n=26\end{array}$ & $\begin{array}{c}\text { Asynchrony Index } \\
\geq 10 \%\end{array}$ & $P$ \\
& $45(33-59)$ & $48(40-58)$ & .79 \\
\hline Age & 80.8 & 66.7 & .39 \\
Male, \% & 30.8 & 22.2 & .62 \\
Penetrating injury, \% & $21(17-29)$ & $22(21-25)$ & .82 \\
Injury Severity Scale score & $2.5(0-8)$ & $2(0-3)$ & .63 \\
Chest Abbreviated Injury & & & \\
$\quad$ Scale score & 43.5 & 16.7 & .23 \\
History of smoking, \% & 16.7 & 14.3 & .88 \\
$\mathrm{COPD} \%$ & $7.38(7.35-7.42)$ & $7.41(7.32-7.44)$ & .97 \\
$\mathrm{pH}$ & $283(254-350)$ & $395(200-468)$ & .57 \\
$\mathrm{P}_{\mathrm{aO}} / \mathrm{F}_{\mathrm{IO}_{2}}$, mm Hg & $43(38-47)$ & $38(35-43)$ & .14 \\
$\mathrm{P}_{\mathrm{aCO}_{2}}$ & $25(22-27)$ & $23(23-26)$ & .34 \\
$\mathrm{HCO}_{3}^{-}$ & & & \\
&
\end{tabular}

Values are median (IQR) unless otherwise specified.

Table 2. Ventilator Settings, Sedation, and Delirium

\begin{tabular}{|c|c|c|c|}
\hline & $\begin{array}{l}\text { Asynchrony } \\
\text { Index } \\
\quad<10 \% \\
n=26\end{array}$ & $\begin{array}{l}\text { Asynchrony } \\
\text { Index } \\
\begin{array}{l}\geq 10 \% \\
n=9\end{array}\end{array}$ & $P$ \\
\hline SIMV + PSV, $\%$ & 38.5 & 100 & .002 \\
\hline Tidal volume, $\mathrm{mL}$ & $600(550-600)$ & $550(550-600)$ & .63 \\
\hline Inspiratory time, $\mathrm{s}$ & $1.15(0.9-1.25)$ & $1.2(0.9-1.2)$ & .86 \\
\hline $\begin{array}{l}\text { Peak inspiratory pressure, } \\
\qquad \mathrm{cm} \mathrm{H}_{2} \mathrm{O}\end{array}$ & $22(16.5-25)$ & $19(17-26)$ & .94 \\
\hline Pressure support, $\mathrm{cm} \mathrm{H}_{2} \mathrm{O}$ & $10(10-10)$ & $10(10-10)$ & .48 \\
\hline PEEP, $\mathrm{cm} \mathrm{H}_{2} \mathrm{O}$ & $5(5-8)$ & $5(5-5)$ & .46 \\
\hline Ventilator rate, breaths/min & $0(0-6)$ & $12(8-14)$ & $<.001$ \\
\hline Patient rate, breaths/min & $12(9-14)$ & $4(3-8)$ & .007 \\
\hline $\begin{array}{l}\text { SIMV with ventilator rate } \\
\geq 10 \text { breaths/min, } \%\end{array}$ & 14.3 & 85.7 & .02 \\
\hline \multicolumn{4}{|l|}{ Fentanyl dosage, $\mu \mathrm{g} / \mathrm{kg}$} \\
\hline In previous hour & $1.01(0.45-1.35)$ & $1.00(0-1.25)$ & .51 \\
\hline In previous 24 hours & $13.8(6.8-29.7)$ & $16.0(2.9-21.2)$ & .61 \\
\hline \multicolumn{4}{|l|}{ Propofol, $\mathrm{mg} / \mathrm{kg}$} \\
\hline In previous hour & $1.35(0-2.4)$ & $0.30(0-1.5)$ & .29 \\
\hline In previous 24 hours & $20.5(5.2-41.6)$ & $10.6(9.5-20.9)$ & .35 \\
\hline RASS score of -1 to $1, \%$ & 61.5 & 33.3 & .31 \\
\hline CAM-ICU positive, $\%$ & 33.3 & 40.0 & .78 \\
\hline \multicolumn{4}{|c|}{$\begin{array}{l}\text { Values are median (IQR) unless otherwise specified. } \\
\text { SIMV = synchronized intermittent mandatory ventilation } \\
\text { PSV = pressure support ventilation } \\
\text { RASS = Richmond Agitation Sedation Scale } \\
\text { CAM-ICU = Confusion Assessment Method for the ICU }\end{array}$} \\
\hline
\end{tabular}

one subject had $75 \%$ of the total of this breath type. Additionally, this subject had a higher set ventilator rate in SIMV than any other subject in the study, which is an associated risk factor for high asynchrony. In light of this finding, we re-analyzed asynchronous breath type proportions excluding this subject. With this datum excluded, the
Table 3. Proportions of Asynchronous Breath Types

\begin{tabular}{lccc}
\hline \hline & Asynchrony & Asynchrony & \\
& Index & Index & \\
& $<10 \%$ & $\geq 10 \%$ & \\
& $n=26$ & $n=9$ & \\
& 4,549 & 10,896 & \\
\hline Total number of breaths & 235 & 1,184 & \\
Total number of asynchronous breaths & & & .01 \\
Asynchronous breath type, no. (\%) & $32(13.6)$ & $120(10.1)$ & \\
Missed/ineffective trigger & $41(17.4)$ & $107(9.0)$ & \\
Double-trigger & $6(2.6)$ & $0(0.0)$ & \\
Short-cycle & $27(11.5)$ & $647(54.7)$ & \\
Long-cycle & $129(54.9)$ & $310(26.2)$ & \\
Ventilator breath stacking & & & \\
\hline
\end{tabular}

Table 4. Outcomes

\begin{tabular}{lccc}
\hline \hline & $\begin{array}{c}\text { Asynchrony Index } \\
<10 \% \\
n=26\end{array}$ & $\begin{array}{c}\text { Asynchrony Index } \\
\geq 10 \%\end{array}$ & $P$ \\
& $7(3-14)$ & $9(4-22)$ & .42 \\
& $11(6-18)$ & $13(8-26)$ & .28 \\
Ventilator days & $17(11-24)$ & $22(10-27)$ & .46 \\
ICU stay, d & 38.5 & 11.1 & .13 \\
Hospital stay, d & 3.9 & 11.1 & .42 \\
Discharged home, \% & & & \\
Mortality, \% & & \\
Values are median (IQR) unless otherwise specified. & & \\
\end{tabular}

difference in prolonged-cycle breaths between the low and high asynchrony groups was still statistically significant $(P<.01)$ but comprised only $23.3 \%$ of the total asynchronous breaths, instead of the $54.7 \%$ shown in Table 3 . Nonetheless, prolonged-cycle breaths are still a significant source of asynchronous breaths in trauma patients.

Unique to this study was the identification of what we termed ventilator breath stacking. This phenomenon occurred during mandatory ventilator breaths in SIMV using the dual control feature (AutoFlow) on the Dräger XL ventilator. When this feature is activated, the software measures dynamic respiratory-system compliance on a breath-to-breath basis, and calculates the pressure required to reach the target tidal volume on the next mandatory breath. Functionally, this mode is pressure limited, but uses a feedback loop to target an inspired tidal volume. The breath type is pressure controlled with decelerating flow. By definition, the ventilator algorithm synchronizes the SIMV mandatory breaths with the patient's spontaneous breaths, to eliminate stacking a mandatory breath on top of a spontaneous one, resulting in a delivered volume much larger than set. We observed that with AutoFlow, synchronization of the mandatory breath and patient effort was not always obtained. Even though the mandatory breath is delivered on top of the spontaneous breath, since the breaths are pressure limited, over-inflation might be avoided. Though the delivered tidal volume is not affected, this phenomenon increases inspiratory time, which is often longer than neural 


\section{Patient-Ventilator Asynchrony in a Traumatically Injured Population}

inspiratory time of spontaneous breaths and may result in asynchrony.

Our study has several limitations. Though the use of ventilator waveforms has been previously validated, we did not utilize esophageal pressure monitoring for the determination of triggering events. As such, an inherent lack of sensitivity for detection may exist. Even with a predetermined window (first $48 \mathrm{~h}$ after intubation) for waveform recording performed over $30 \mathrm{~min}$, periods of asynchrony could be missed, thus underestimating the prevalence of this phenomenon in our population. Furthermore, recordings occurred during daytime hours and thus might have omitted asynchronous periods at night, when interruptions in sleep by normal ICU care may account for periods of agitation and/or delirium. Though not statistically significant, a trend toward RASS scores outside of -1 to 1 was associated with those with a higher asynchrony index. The small sample size of this study may have contributed to false conclusions in regard to sedation differences between the groups. We used the Dräger XL ventilator exclusively for this study, due to its compatibility with the recording software. We assume that other ICU ventilators would produce similar patient-ventilator interactions using the same modes. Finally, this study was conducted in a single institution, with 35 trauma subjects, using various modes of mechanical ventilation. Our study may not have had the power to find an outcome difference between the 2 groups, so a larger cohort of trauma subjects with uniformity in ventilation mode, over multiple institutions, may support alternative findings. Future work may focus on a larger population of trauma patients, compared both internally and externally to both surgical and non-surgical cohorts.

\section{Conclusions}

Patient-ventilator asynchrony is common in mechanically ventilated traumatically injured patients. However, there were no significant associations between high and low asynchrony and ventilator days, ICU and hospital stay, and mortality. The most common ventilator mode associated with high asynchrony in our subjects was SIMV with a set frequency of $\geq 10$ breaths/min. While ineffective efforts have represented as much as $85 \%$ of asynchronous breaths in medical patients, this was not observed in our cohort of surgical subjects. Careful matching of the appropriate ventilator mode(s) and settings to the patient's ventilatory needs may help minimize asynchrony and improve patient comfort.

\section{REFERENCES}

1. Thille AW, Rodriguez P, Cabello B, Lellouche F, Brochard L. Patient-ventilator asynchrony during assisted mechanical ventilation. Intensive Care Med 2006;32(10):1515-1522.

2. Sassoon CS, Foster GT. Patient-ventilator asynchrony. Curr Opin Crit Care 2001;7(1):28-33.

3. Tobin MJ, Jubran A, Laghi F. Patient-ventilator interaction. Am J Respir Crit Care Med 2001;163(5):1059-1063.

4. Parthasarathy S, Jubran A, Tobin MJ. Cycling of inspiratory and expiratory muscle groups with the ventilator in airflow limitation. Am J Respir Crit Care Med 1998;158(5):1471-1478.

5. Sassoon, CSH. Triggering of the ventilator in patient-ventilator interactions. Respir Care 2011;56(1):39-51.

6. Chao DC, Stearn-Hassenpflug M. Patient-ventilator trigger asynchrony in prolonged mechanical ventilation. Chest 1997;112(6):1592-1599.

7. Vitacca M, Bianchi L, Zanotti E, Vianello A, Barbano L, Porta R, Clini E. Assessment of physiologic variables and subjective comfort under different levels of pressure support ventilation. Chest 2004; 126(3):851-859.

8. de Wit M, Pedram S, Best AM, Epstein S. Observational study of patient-ventilator asynchrony and relationship to sedation level. J Crit Care 2009;24(1):74-80.

9. Baker SP, O'Neill B, Haddon W Jr, Long WB. The injury severity score: a method for describing patients with multiple injuries and evaluating emergency care. J Trauma 1974;14(3):187-196.

10. Sessler CN, Gosnell MS, Grap MJ, Brophy GM, O'Neal PV, Keane $\mathrm{KA}$, et al. The Richmond Agitation-Sedation Scale: validity and reliability in adult intensive care unit patients. Am J Respir Crit Care Med 2002;166(10):1338-1344.

11. Ely EW, Margolin R, Francis J, May L, Truman B, Dittus R, et al. Evaluation of delirium in critically ill patients: validation of the Confusion Assessment Method for the Intensive Care Unit (CAMICU). Crit Care Med 2001;29(7):1370-1379.

12. Robinson BR, Mueller EW, Henson K, Branson RD, Barsoum S, Tsuei BJ. An analgesia-delirium-sedation protocol for critically ill trauma patients reduces ventilator days and hospital length of stay. J Trauma 2008;65(3):517-526.

13. Beck J, Gottfried SB, Navalesi P, Skrobik Y, Comtois N, Rossini M, Sinderby C. Electrical activity of the diaphragm during pressure support ventilation in acute respiratory failure. Am J Respir Crit Care Med 2001;164(3):419-424.

14. Leung P, Jubran A, Tobin MJ. Comparison of assisted ventilator modes on triggering, patient effort, and dyspnea. Am J Respir Crit Care Med 1997;155(6):1940-1948.

15. Nava S, Bruschi C, Rubini F, Palo A, Iotti G, Braschi A. Respiratory response and inspiratory effort during pressure support ventilation in COPD patients. Intensive Care Med 1995;21(11):871-879.

16. Fabry B, Guttmann J, Eberhard L, Bauer T, Haberthür C, Wolff. An analysis of desynchronization between the spontaneously breathing patient and ventilator during inspiratory pressure support. Chest 1995;107(5):1387-1394.

This article is approved for Continuing Respiratory Care Education credit. For information and to obtain your CRCE

(free to AARC members) visit

www.rcjournal.com

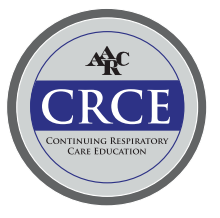

